complex. A detailed description of the observations, results, and models are given in Sofue et al. (1986).

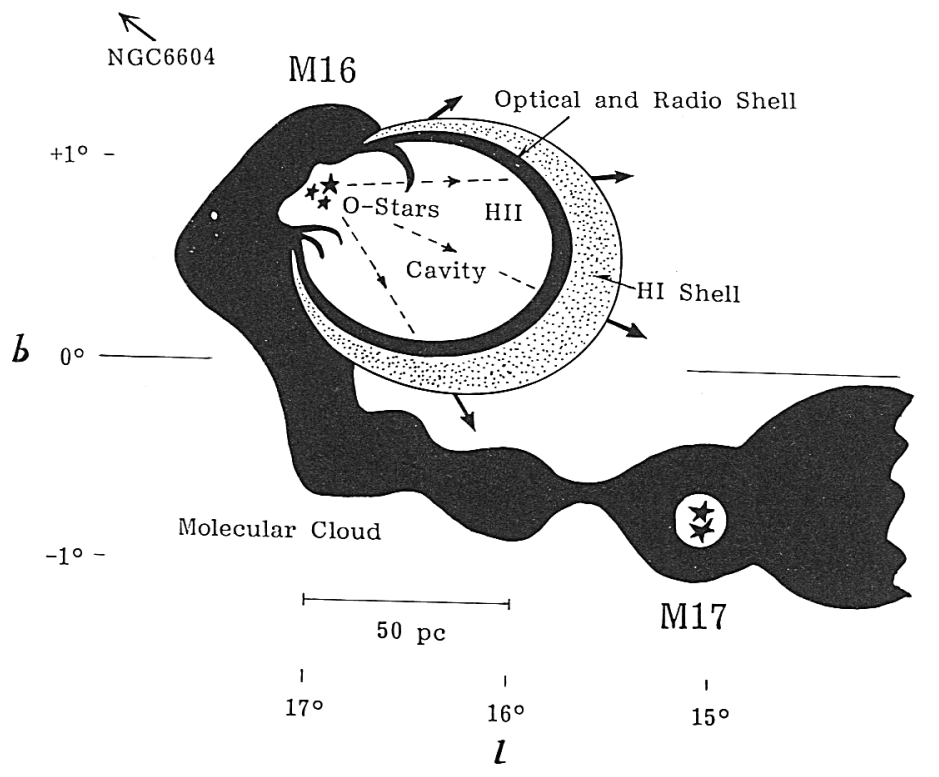

Fig. 2. Schematic illustration of the relationship of the optical, radio and $\mathrm{HI}$ shells to the star-forming site M16 and M17 in the large molecular cloud complex.

\title{
REFERENCES
}

Sofue, Y., Handa, T., Fürst, E., Reich, W, and Reich, P.: 1968, Pub1. Astron. Soc. Japan, in press.

Tomisaka, K., Habe, A., and Ikeuchi, S.: 1981, Astrophys. Sp. Sci. 78, 273.

S201: THE IONIZED SKIN OF A MOLECULAR BLOB

M. Fel1i, R. Cesaroni

Osservatorio di Arcetri, Florence, Italy

R.M. Hjellming

N.R.A.O. USA

S201 is an optically visible HII region whose ionizing star is hidden to the sight by heavy obscuration. The radio continuum surface 
brightness distribution observed at $6-\mathrm{cm}$ with the VLA can be described by two distinct components: a) an extended diffuse structure, which closely reproduces the shape of the observable nebulosity, and b) a sharp bright arc feature on the east side of the extended component, unresolved towards the outside at the resolution of $\sim 4 "$.

From CO maps of the same region we know that on the east side the nebula is bounded by a high density molecular cloud (see also Wramdemark, Lynga, and Johansson, paper presented at this symposium). To reproduce the surface brightness observed in the radio continuum, we have used a three dimensional model in which the ionized gas distribution is determined by gas outflow from a spherical molecular blob. The ionization is produced by an early type star placed outside the blob. The whole complex is also surrounded by a low density diffuse region.

To derive the electron density distribution we made the following assumptions: a) at the ionization front (assumed to be spherical) the $\mathrm{n}_{\mathrm{e}}$ is determined by the stellar ionizing flux reaching the surface, which, in turn, is determined by geometrical factors and by the gas absorption along the path, and b) the ionized gas is assumed to expand radially at constant velocity and to be at constant temperature.

In addition to the ne produced by the erosion of the molecular cloud, a "standard" Strömgren region due to the diffuse surrounding component is also added.

This simple model can fit the observed surface brightness distribution quite well. In particular, the required UV photon flux is $8.4 \times$ $10^{44} \mathrm{~s}^{-1}$ (equivalent to an 09 ZAMS) of which only a fraction is absorbed in the outflow. The remaining UV photons are absorbed in the diffuse component. The distance of the exciting star from the molecular blob (assumed to have a radius of $0.3 \mathrm{pc}$ ) is $0.34 \mathrm{pc}$. It is remarkable that the position of the exciting star derived in this way closely matches that of a 2 micron source (Manpaso, private communication) which is the proposed candidate for the ionizing star of the nebula.

The maximum electron density of the ionization front turns out to be $1.1 \times 10^{3} \mathrm{~cm}^{-3}$ and the erosion of the molecular blob due to the ionization is $4 \times 10^{-5} \mathrm{M}_{\odot} \mathrm{yr}^{-1}$. If this rate has been constant since the turn on of the early type star, the total mass of the diffuse HII region can be produced by the erosion over a time scale of $5 \times 10^{5}$ years.

\section{EXPANSION OF ULTRA-COMPACT H II REGIONS}

J.M. Moran, M.J. Reid, B. Sams

Harvard-Smithsonian Center for Astrophysics, USA

The dynamical properties of the ionized envelopes surrounding massive, newly formed stars can be probed by the measurement of radio recombination lines over a wide range of frequencies. In an expanding 